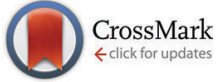

Cite this: Phys. Chem. Chem. Phys., 2016, 18, 27396

Received 21st June 2016, Accepted 30th August 2016 DOI: $10.1039 / c 6 c p 04347 j$

www.rsc.org/pccp

\section{Modeling blue to UV upconversion in $\beta-\mathrm{NaYF}_{4}: \mathrm{Tm}^{3+}{ }_{\dagger}$}

\author{
Pedro Villanueva-Delgado, ${ }^{* a}$ Karl W. Krämer, ${ }^{a}$ Rafael Valiente, ${ }^{b}$ Mathijs de Jong ${ }^{c}$ \\ and Andries Meijerink ${ }^{C}$
}

Samples of $0.01 \%$ and $0.3 \% \mathrm{Tm}^{3+}$-doped $\beta-\mathrm{NaYF}_{4}$ show upconverted UV luminescence at $27660 \mathrm{~cm}^{-1}$ $(361 \mathrm{~nm})$ after blue excitation at $21140 \mathrm{~cm}^{-1}(473 \mathrm{~nm})$. Contradictory upconversion mechanisms in the literature are reviewed and two of them are investigated in detail. Their agreement with emission and two-color excitation experiments is examined and compared. Decay curves are analyzed using the Inokuti-Hirayama model, an average rate equation model, and a microscopic rate equation model that includes the correct extent of energy transfer. Energy migration is found to be negligible in these samples, and hence the average rate equation model fails to correctly describe the decay curves. The microscopic rate equation model accurately fits the experimental data and reveals the strength and multipolarity of various interactions. This microscopic model is able to determine the most likely upconversion mechanism.

\section{Introduction}

Upconversion (UC) is the absorption of two or more low-energy photons and the subsequent emission of a high-energy photon. ${ }^{1}$ Several trivalent lanthanide ions are commonly used for this purpose, for example singly doped or codoped into insulator lattices. $\mathrm{Tm}^{3+}$ ions can emit light at different wavelengths from the NIR to the UV and show upconversion in singly doped samples under NIR, red, and blue excitation. ${ }^{2-5} \mathrm{Tm}^{3+}$ codoped with $\mathrm{Yb}^{3+}$ also shows particularly strong blue and UV emission under $980 \mathrm{~nm}$ excitation. ${ }^{6-8}$

There are different applications of upconversion phosphors, for example in biomedical research, ${ }^{9}$ dental medicine, ${ }^{10}$ photovoltaic energy production, ${ }^{11}$ and photocatalysis. ${ }^{12,13}$

The detailed mechanism of singly doped $\mathrm{Tm}^{3+}$ upconversion under different excitation energies has been studied in several crystalline and vitreous environments. However, the actual processes involved are not clear for the case of blue excitation that results in UV emission. ${ }^{2}$ These processes may also be responsible for the efficient blue and UV emission in samples codoped with $\mathrm{Yb}^{3+}$.

There are several energy transfer (ET) processes that lead to upconversion. In this work we will examine two of them: energy

\footnotetext{
${ }^{a}$ Department of Chemistry and Biochemistry, University of Bern, 3012 Bern, Switzerland. E-mail: pedro.villanueva@dcb.unibe.ch

${ }^{b}$ Departamento de Física Aplicada, Facultad de Ciencias, Universidad de Cantabria-IDIVAL, 39005 Santander, Spain

${ }^{c}$ Condensed Matter and Interfaces, Debye Institute for Nanomaterials Science, Utrecht University, 3508TA Utrecht, The Netherlands

$\dagger$ Electronic supplementary information (ESI) available. See DOI: 10.1039/ с6ср04347j
}

transfer upconversion (ETU) and cross-relaxation (CR). Both processes have the same requirements: two ions in close proximity and resonance between energy states (spectral overlap).

Two kinds of ET interactions are possible, multipolar or exchange, depending on the distance between the ions and the electronic orbital overlap. ${ }^{14}$ For the $\beta-\mathrm{NaYF}_{4}: \mathrm{Tm}^{3+}$ samples investigated in this paper, the exchange interaction is not significant as the minimum distance between rare earth ions in $\beta-\mathrm{NaYF}_{4}$ is $3.53 \AA$ and the low doping increases the average first neighbor distance to more than $10 \AA^{15,16}$ In the multipolar case, the strength of the interaction $W$ can be expanded as a series of inverse powers of the distance $R$ between the sensitizer $(\mathrm{S})$ and the activator $(\mathrm{A}):^{17}$

$$
W(R)=\frac{C_{\mathrm{SA}}^{(n)}}{R^{n}},
$$

where $n$ is the multipolarity of the interaction, $n=6,8$, and 10 for dipole-dipole $(\mathrm{d}-\mathrm{d})$, dipole-quadrupole $(\mathrm{d}-\mathrm{q})$, and quadrupole-quadrupole $(\mathrm{q}-\mathrm{q})$, respectively, and $C_{\mathrm{SA}}^{(n)}$ is a constant that depends on the multipolarity. The dominant multipolarity of a transition depends on the symmetry of the states involved. ${ }^{14,18}$

Several models have been developed to predict the decay of a sensitizer in the presence of an activator under different assumptions. The Inokuti-Hirayama model, for example, predicts the decay curve of a sensitizer that can decay radiatively or transfer its energy to an activator situated nearby. Other interactions such as energy migration among the sensitizers or ET to excited states of the activator (ETU or CR) are not taken into account. ${ }^{19}$ The random lattice positions of the activator and the 
combination of the two relaxation pathways produce a nonexponential decay, especially at the beginning of the decay curve. The beginning of the curve is mostly affected by the ET to the activator, while the tail of the curve reproduces the single exponential decay of the sensitizer. The analytical form is given by

$$
I(t)=I_{0} \exp \left(\frac{-t}{\tau_{0}}-\gamma\left(\frac{t}{\tau_{0}}\right)^{\frac{3}{n}}\right)+B
$$

where $I_{0}$ is the initial luminescence intensity, $B$ is the luminescence background, $\tau_{0}$ is the intrinsic lifetime of the sensitizer state, $\gamma$ is the energy transfer parameter, and $n$ is the multipolarity of the interaction. The ET parameter $\gamma$ is related to the activator concentration $c_{\mathrm{A}}$ and the critical radius $R_{\mathrm{c}}$ (the distance at which the intrinsic decay and ET rates are equal):

$$
\gamma=\frac{4 \pi}{3} c_{\mathrm{A}} R_{\mathrm{c}}^{3} \Gamma\left(1-\frac{3}{n}\right),
$$

where $\Gamma$ is the gamma function with values $\Gamma(1 / 2)=1.7725$, $\Gamma(5 / 8)=1.4345$, and $\Gamma(7 / 10)=1.2981$, for $\mathrm{d}-\mathrm{d}$, $\mathrm{d}-\mathrm{q}$, and $\mathrm{q}-\mathrm{q}$ interactions, respectively. The sensitizer-activator transfer rate in eqn (1) is related to the critical radius

$$
C_{\mathrm{SA}}^{(n)}=\frac{1}{\tau_{0}} R_{\mathrm{c}}{ }^{n}
$$

Models by Zusman, ${ }^{20,21}$ Burshtein, ${ }^{22,23}$ and Yokota-Tanimoto ${ }^{24,25}$ include energy migration in their treatment. A model by Grant, based on rate equations, can predict not only the decay of the sensitizer, but also the decay of the activator. ${ }^{26}$ However this model assumes infinite energy migration. A modification by Zubenko accounts for a finite speed of energy migration, but it is difficult to extend it with more interactions. ${ }^{27,28}$

A recent microscopic rate equation model includes the effect of the crystalline environment (the distances between the ions) and the finite speed of energy migration. ${ }^{16}$ This model can predict the dynamics of a system at different dopant concentrations and excitation powers. A fit to experimental data provides the most likely critical radius and multipolarity of the different interactions.

\section{Experimental methods}

Microcrystalline powder samples of $\beta-\mathrm{NaYF}_{4}: 0.3 \% \mathrm{Tm}^{3+}$ and $\beta-\mathrm{NaYF}_{4}: 0.01 \% \mathrm{Tm}^{3+}$ were synthesized as described in ref. 29.

Visible and NIR emission spectra and decay curves were recorded using an Opotek Opolette $\mathrm{HE}$ 355II laser as an excitation source with a pulse width of $7 \mathrm{~ns}$ and a repetition rate of $10 \mathrm{~Hz}$. An Edinburgh Instruments FLS920 spectrophotometer with a $0.3 \mathrm{~m}$ single emission monochromator and Hamamatsu R928 UV/VIS or R5509-73 NIR photomultiplier tubes (PMTs) were used to detect the light. An Edinburgh Instruments PCS900 single-photon counter and multichannel analyzer were used to record the decay curves.

The two-color pump and probe excitation experiments were carried out using an Opotek Opolette HE 355II (7 ns pulse) and an Ekspla NT342B-10-SH/DUV OPO (5 ns pulse) as excitation sources (both with $10 \mathrm{~Hz}$ repetition rate), a $0.55 \mathrm{~m}$ TRIAX 550 single emission monochromator, and a Hamamatsu R928 PMT. The signal was analyzed using a Stanford Research SR400 gated photon counter. Both laser pulses were synchronized using a Standford Research DG535 pulse generator to arrive at the sample at the same time. The UV upconversion emission spectra were recorded using the same setup but using only the Ekspla laser as an excitation source.

All measurements were performed at room temperature.

\section{Results}

The powder X-ray diffractograms of the $\beta-\mathrm{NaYF}_{4}: 0.01 \% \mathrm{Tm}^{3+}$ and $\beta-\mathrm{NaYF}_{4}: 0.3 \% \mathrm{Tm}^{3+}$ samples correspond to the pure hexagonal $\beta-\mathrm{NaREF}_{4}$ phase ( $\mathrm{RE}=$ rare earth ion) that crystallizes in the space group $P \overline{6}$. The $\mathrm{RE}^{3+}$ sites with $C_{1}$ and $C_{3 \mathrm{~h}}$ symmetry have both a nine-fold coordination by $\mathrm{F}^{-}$ions and are randomly occupied by $\mathrm{Y}^{3+}$ and $\mathrm{Tm}^{3+}$ ions, ${ }^{15}$ see Fig. $\mathrm{S} 1$, ESI. $\dagger$

Fig. 1 shows the emission spectra of $\beta-\mathrm{NaYF}_{4}$ doped with $0.3 \%$ and $0.01 \% \mathrm{Tm}^{3+}$ after ${ }^{3} \mathrm{H}_{6} \rightarrow{ }^{1} \mathrm{G}_{4}$ excitation at $21140 \mathrm{~cm}^{-1}$ $(473 \mathrm{~nm})$. Both samples have been investigated under the same experimental conditions. The peaks have been assigned to $f-f$ transitions of $\mathrm{Tm}^{3+}$ based on their lifetimes and energies, according to the Dieke diagram. ${ }^{30}$

The low energy side of the spectra shows emission from the ${ }^{1} \mathrm{G}_{4},{ }^{3} \mathrm{H}_{4}$, and ${ }^{3} \mathrm{~F}_{4}$ states to the ${ }^{3} \mathrm{H}_{6}$ ground state and other intermediate states. The spectra are normalized to the ${ }^{1} \mathrm{G}_{4} \rightarrow{ }^{3} \mathrm{~F}_{4}$ emission intensity. The major differences observed between the two samples are the ${ }^{3} \mathrm{H}_{4} \rightarrow{ }^{3} \mathrm{H}_{6}$ and ${ }^{1} \mathrm{G}_{4} \rightarrow{ }^{3} \mathrm{H}_{4}$ emission intensities. The former emission is about 2.5 times stronger in the $0.3 \%$ sample than in the $0.01 \%$ sample, the latter however is 2 times weaker. These differences are discussed in Section 4.

The small peak close to $6200 \mathrm{~cm}^{-1}$ (numbered 1 in Fig. 1) is assigned to the ${ }^{3} \mathrm{~F}_{4} \rightarrow{ }^{3} \mathrm{H}_{6}$ transition. Since the detector efficiency strongly decreases towards that end of the spectrum, that peak's position and intensity can only be taken as indicative.

The band centered at $6780 \mathrm{~cm}^{-1}$ (numbered 2 and 4 in Fig. 1) is assigned to the transitions ${ }^{3} \mathrm{H}_{4} \rightarrow{ }^{3} \mathrm{~F}_{4}$ and ${ }^{1} \mathrm{G}_{4} \rightarrow{ }^{3} \mathrm{~F}_{3}$. The different peaks in the band have decay curves that correspond to the ${ }^{3} \mathrm{H}_{4}$ or ${ }^{1} \mathrm{G}_{4}$ states, see Fig. $2 \mathrm{~b}$ and c. Most of the band intensity originates from the ${ }^{3} \mathrm{H}_{4} \rightarrow{ }^{3} \mathrm{~F}_{4}$ transition, see Fig. 3a, and also Fig. S2, ESI. $\dagger$

The high energy side of the spectra was recorded using a different detector and the intensities cannot be compared with the low energy side. The emission intensity of the $0.01 \%$ sample is 50 times weaker than that of the $0.3 \%$ sample. Aside from the ${ }^{1} \mathrm{D}_{2} \rightarrow{ }^{3} \mathrm{H}_{6}$ emission, a peak corresponding to the ${ }^{3} \mathrm{P}_{0} \rightarrow{ }^{3} \mathrm{~F}_{4}$ emission is present in the spectra. The mechanism responsible for the population of the ${ }^{3} \mathrm{P}_{0}$ state is beyond the scope of this paper. An ETU process from the ${ }^{1} \mathrm{D}_{2}$ or ${ }^{1} \mathrm{G}_{4}$ states is likely, as an ESA process from the ${ }^{1} \mathrm{G}_{4}$ state is not resonant with any $\mathrm{Tm}^{3+}$ state.

Fig. 2 shows the decay curves of the ${ }^{1} \mathrm{D}_{2} \rightarrow{ }^{3} \mathrm{H}_{6},{ }^{1} \mathrm{G}_{4} \rightarrow{ }^{3} \mathrm{~F}_{4}$, and ${ }^{3} \mathrm{H}_{4} \rightarrow{ }^{3} \mathrm{~F}_{4}$ transitions in $\beta-\mathrm{NaYF}_{4}: 0.3 \% \mathrm{Tm}^{3+}$. The reason that the ${ }^{1} \mathrm{G}_{4} \rightarrow{ }^{3} \mathrm{H}_{6}$ decay is not shown here is due to the 


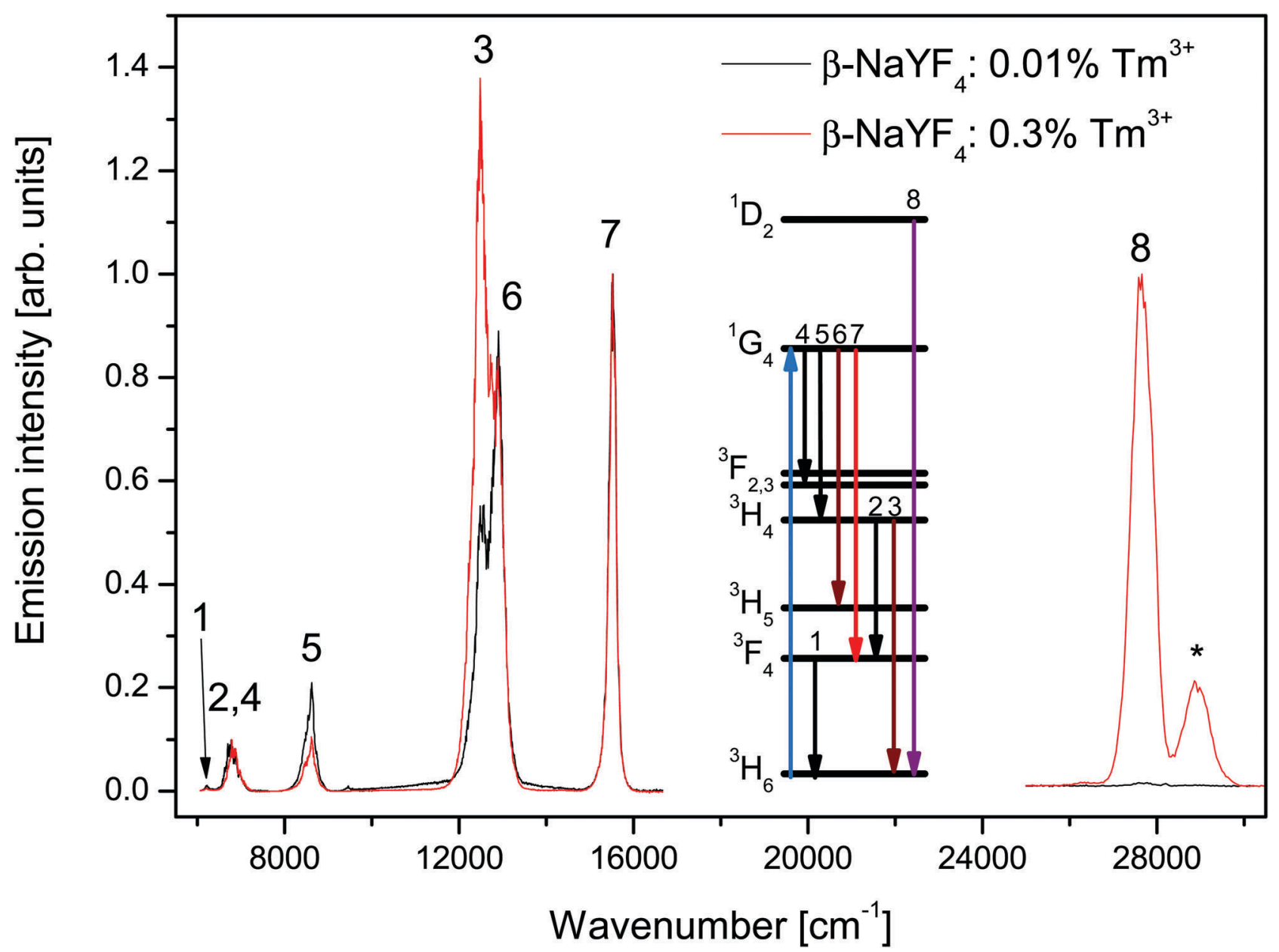

Fig. 1 Emission spectra of $\beta-\mathrm{NaYF}_{4}$ doped with $0.3 \% \mathrm{Tm}^{3+}$ (red line) and $0.01 \% \mathrm{Tm}^{3+}$ (black line) after ${ }^{3} \mathrm{H}_{6} \rightarrow{ }^{1} \mathrm{G}_{4}$ excitation at $21140 \mathrm{~cm}^{-1}$. The low energy side is normalized to the ${ }^{1} \mathrm{G}_{4} \rightarrow{ }^{3} \mathrm{~F}_{4}$ emission intensity. The high energy side $\left({ }^{1} \mathrm{D}_{2} \rightarrow{ }^{3} \mathrm{H}_{6}\right)$ was recorded using a different detector, but otherwise with the same settings for both samples. The band marked with an asterisk (*) corresponds to the $\mathrm{Tm}^{3+}{ }^{3} \mathrm{P}_{0} \rightarrow{ }^{3} \mathrm{~F}_{4}$ emission.

difficulty of measuring its emission close to the excitation energy. The ${ }^{3} \mathrm{H}_{4} \rightarrow{ }^{3} \mathrm{~F}_{4}$ decay has been chosen instead of the ${ }^{3} \mathrm{H}_{4} \rightarrow{ }^{3} \mathrm{H}_{6}$ because the latter is very close in energy to the ${ }^{1} \mathrm{G}_{4} \rightarrow{ }^{3} \mathrm{H}_{5}$ emission and its rise time is partially masked by it. All decay curves from the ${ }^{1} \mathrm{G}_{4}$ and ${ }^{3} \mathrm{H}_{4}$ states are shown in Fig. S4, ESI. $\dagger$

The ${ }^{1} \mathrm{G}_{4} \rightarrow{ }^{3} \mathrm{~F}_{4}$ decay in Fig. 2b is not single exponential, which is evidence of energy transfer. The tail decay time is $\tau_{\mathrm{d}}=719 \mu \mathrm{s}$, which is shorter than the intrinsic lifetime $\tau=758 \mu \mathrm{s}$, see Fig. S3b, ESI. $\dagger$ The most salient feature in Fig. $2 \mathrm{a}$ and $\mathrm{c}$ is the fast rise time of $\tau_{\mathrm{r}}=19 \mu \mathrm{s}$ and $\tau_{\mathrm{r}}=96 \mu \mathrm{s}$, respectively. The decay of the ${ }^{1} \mathrm{D}_{2} \rightarrow{ }^{3} \mathrm{H}_{6}$ transition is not single exponential with fast and slow decay components of $\tau_{\mathrm{d}, 1}=$ $161 \mu \mathrm{s}$ and $\tau_{\mathrm{d}, 2}=416 \mu \mathrm{s}$, respectively. Both decay times are slower than the intrinsic ${ }^{1} \mathrm{D}_{2}$ lifetime of $\tau=67.5 \mu \mathrm{s}$, see Fig. S3a, ESI. $\dagger$ The ${ }^{3} \mathrm{H}_{4} \rightarrow{ }^{3} \mathrm{~F}_{4}$ decay lifetime of $\tau_{\mathrm{d}}=2.015 \mathrm{~ms}$ is very similar to the ${ }^{3} \mathrm{H}_{4} \rightarrow{ }^{3} \mathrm{H}_{6}$ decay lifetime of $1.94 \mathrm{~ms}$ observed for the direct excitation of the ${ }^{3} \mathrm{H}_{4}$ state in the $0.01 \%$ sample at low power, see Fig. S3c, ESI. $\dagger$

The spectral overlap of selected transitions in $\beta-\mathrm{NaYF}_{4}: 0.3 \%$ $\mathrm{Tm}^{3+}$ is presented in Fig. 3. Fig. 3a shows the spectral overlap between the ${ }^{1} \mathrm{G}_{4} \rightarrow{ }^{1} \mathrm{D}_{2}$ excited state absorption, see Fig. S5, ESI, $\uparrow$ and the ${ }^{3} \mathrm{H}_{4} \rightarrow{ }^{3} \mathrm{~F}_{4}$ and ${ }^{1} \mathrm{G}_{4} \rightarrow{ }^{3} \mathrm{~F}_{3}$ emission bands, see Fig. 1 . The strong overlap between the transitions is a necessary condition for the ETU processes ${ }^{3} \mathrm{H}_{4}+{ }^{1} \mathrm{G}_{4} \rightarrow{ }^{3} \mathrm{~F}_{4}+{ }^{1} \mathrm{D}_{2}$ and ${ }^{1} \mathrm{G}_{4}+{ }^{1} \mathrm{G}_{4} \rightarrow{ }^{3} \mathrm{~F}_{3}+{ }^{1} \mathrm{D}_{2}$ between two interacting $\mathrm{Tm}^{3+}$ ions in close proximity.

Fig. 3 b compares the ground state absorption ${ }^{3} \mathrm{H}_{6} \rightarrow{ }^{3} \mathrm{H}_{5}$, see Fig. S6, ESI, $\dagger$ to the ${ }^{1} \mathrm{G}_{4} \rightarrow{ }^{3} \mathrm{H}_{4}$ emission, see Fig. 1 . The overlap is smaller than that in Fig. $3 \mathrm{a}$, but still significant. Thus the CR process ${ }^{3} \mathrm{H}_{6}+{ }^{1} \mathrm{G}_{4} \rightarrow{ }^{3} \mathrm{H}_{5}+{ }^{3} \mathrm{H}_{4}$ is a possible ET process between two nearby $\mathrm{Tm}^{3+}$ ions.

\section{Analysis}

In the literature, contradictory $\mathrm{Tm}^{3+}$ upconversion mechanisms are proposed for the population of the ${ }^{1} \mathrm{D}_{2}$ state after ${ }^{1} \mathrm{G}_{4}$ excitation. Often, one ETU step processes are proposed: ${ }^{1} \mathrm{G}_{4}+{ }^{1} \mathrm{G}_{4} \rightarrow{ }^{3} \mathrm{~F}_{3}+$ ${ }^{1} \mathrm{D}_{2},{ }^{3} \mathrm{~F}_{3}+{ }^{3} \mathrm{~F}_{3} \rightarrow{ }^{3} \mathrm{H}_{6}+{ }^{1} \mathrm{D}_{2},{ }^{6,7}$ or ${ }^{1} \mathrm{G}_{4}+{ }^{1} \mathrm{G}_{4} \rightarrow{ }^{3} \mathrm{H}_{4}+{ }^{1} \mathrm{D}_{2}$. ${ }^{2}$ Those investigations were carried out in different host lattices (including glasses). Samples additionally codoped with $\mathrm{Yb}^{3+}$ are sometimes used, which complicates the analysis. The different crystal-field 

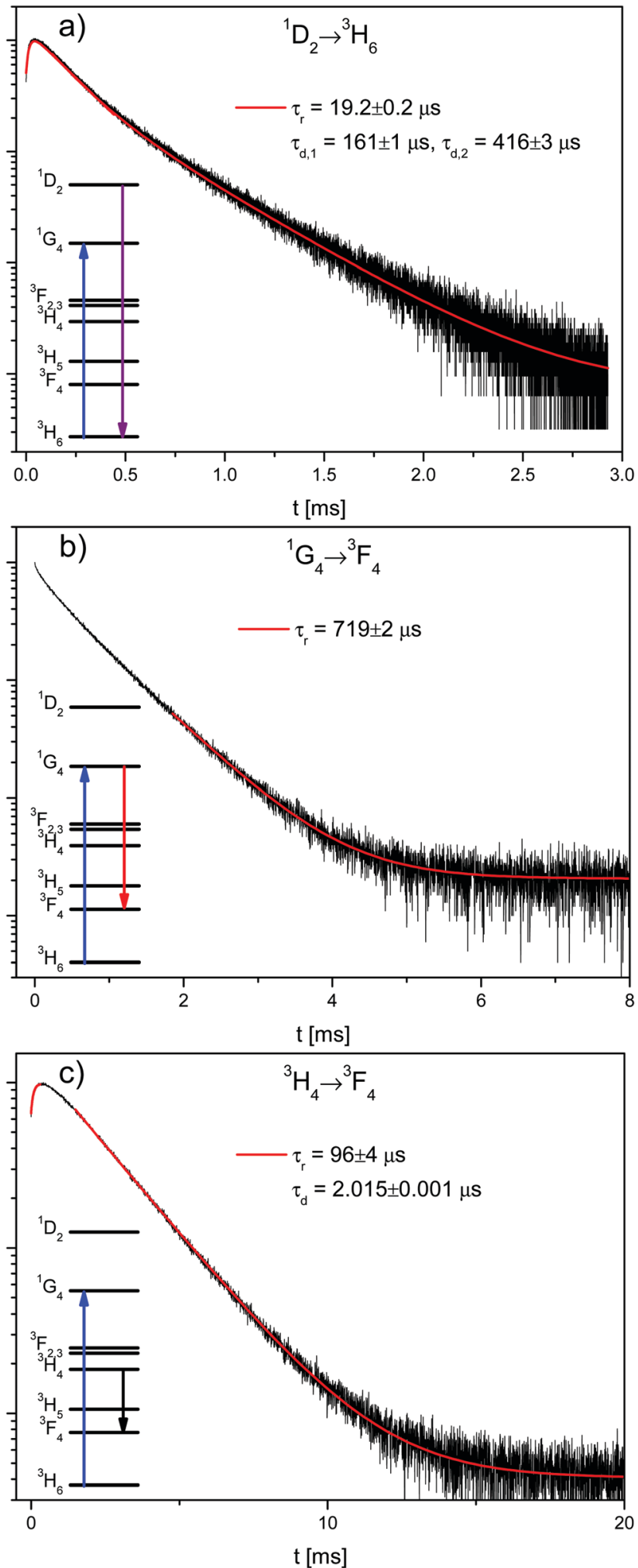

Fig. 2 Decay curves of $\beta-\mathrm{NaYF}_{4}: 0.3 \% \mathrm{Tm}^{3+}$ (black lines) after ${ }^{3} \mathrm{H}_{6} \rightarrow{ }^{1} \mathrm{G}_{4}$ excitation (at $21140 \mathrm{~cm}^{-1}$ ) with exponential rise $\tau_{\mathrm{r}}$ and decay $\tau_{\mathrm{d}}$ fits (red lines). (a) ${ }^{1} \mathrm{D}_{2} \rightarrow{ }^{3} \mathrm{H}_{6}$ transition with a single exponential fit to the rise and a double exponential fit to the decay. (b) ${ }^{1} \mathrm{G}_{4} \rightarrow{ }^{3} \mathrm{~F}_{4}$ transition with a single exponential fit to the decay. (c) ${ }^{3} \mathrm{H}_{4} \rightarrow{ }^{3} \mathrm{~F}_{4}$ transition with single exponential rise and decay fits.
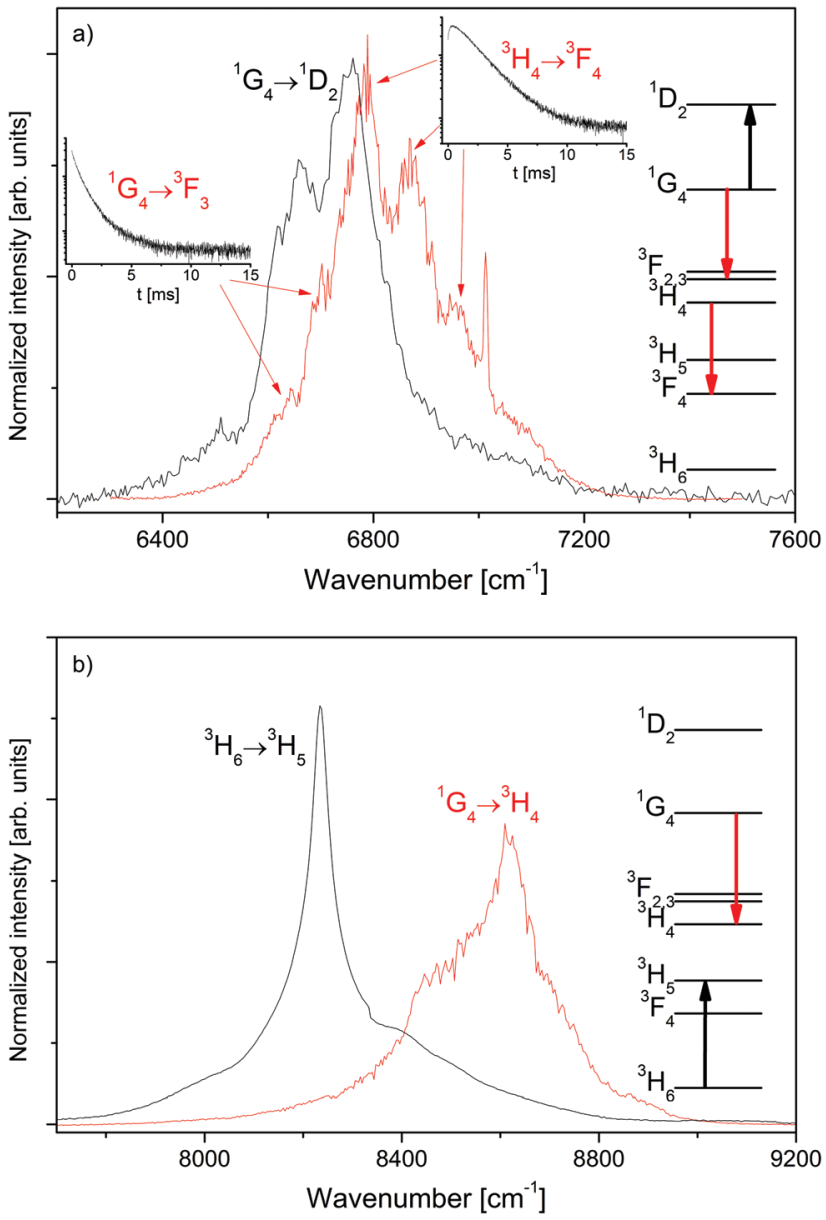

Fig. 3 Spectral overlap (normalized to the area) of selected transitions in $\beta-\mathrm{NaYF}_{4}: 0.3 \% \mathrm{Tm}^{3+}$. (a) ${ }^{1} \mathrm{G}_{4} \rightarrow{ }^{1} \mathrm{D}_{2}$ excited state absorption (black line) and ${ }^{3} \mathrm{H}_{4} \rightarrow{ }^{3} \mathrm{~F}_{4} /{ }^{1} \mathrm{G}_{4} \rightarrow{ }^{3} \mathrm{~F}_{3}$ emissions (red line). (b) ${ }^{3} \mathrm{H}_{6} \rightarrow{ }^{3} \mathrm{H}_{5}$ ground state absorption (black line) and ${ }^{1} \mathrm{G}_{4} \rightarrow{ }^{3} \mathrm{H}_{4}$ emission (red line). The respective transitions are indicated in the $\mathrm{Tm}^{3+}$ energy state diagrams on the right hand side.

environments result in small shifts in energy that may affect the resonance between transitions and thus different mechanisms may be responsible for the ${ }^{1} \mathrm{D}_{2}$ upconversion in different samples. A recent work has studied the role of CR steps in depopulating the ${ }^{1} \mathrm{D}_{2},{ }^{1} \mathrm{G}_{4}$, and ${ }^{3} \mathrm{H}_{4}$ states for downconversion. ${ }^{31}$

We now consider two upconversion mechanisms: schemes I and II, shown in Fig. 4. Scheme I involves two ET processes and three $\mathrm{Tm}^{3+}$ ions. Scheme II involves an ETU process, multiphonon relaxation, and two $\mathrm{Tm}^{3+}$ ions. Both schemes explain the very fast rise times of the ${ }^{1} \mathrm{D}_{2}$ and ${ }^{3} \mathrm{H}_{4}$ states, see Fig. 2a and c.

Scheme I: the ${ }^{1} \mathrm{G}_{4} \rightarrow{ }^{3} \mathrm{H}_{4}$ decay time of $719 \mu \mathrm{s}$ is much slower than the $96 \mu$ s rise time of the ${ }^{3} \mathrm{H}_{4}$ luminescence, see Fig. $2 \mathrm{~b}$ and c. Accordingly, the ${ }^{3} \mathrm{H}_{4}$ state cannot be directly populated by the ${ }^{1} \mathrm{G}_{4}$ state. But energy transfer processes between two ions can affect a state population very quickly; in fact, the CR step ${ }^{3} \mathrm{H}_{6}+{ }^{1} \mathrm{G}_{4} \rightarrow{ }^{3} \mathrm{H}_{5}+{ }^{3} \mathrm{H}_{4}$ is able to populate the ${ }^{3} \mathrm{H}_{4}$ state very rapidly. The emission spectra, see Fig. 1 , support this statement. The spectra of both samples are very similar except for the ${ }^{3} \mathrm{H}_{4} \rightarrow{ }^{3} \mathrm{H}_{6}$ and ${ }^{1} \mathrm{G}_{4} \rightarrow{ }^{3} \mathrm{H}_{4}$ transitions. The ${ }^{3} \mathrm{H}_{4} \rightarrow{ }^{3} \mathrm{H}_{6}$ 


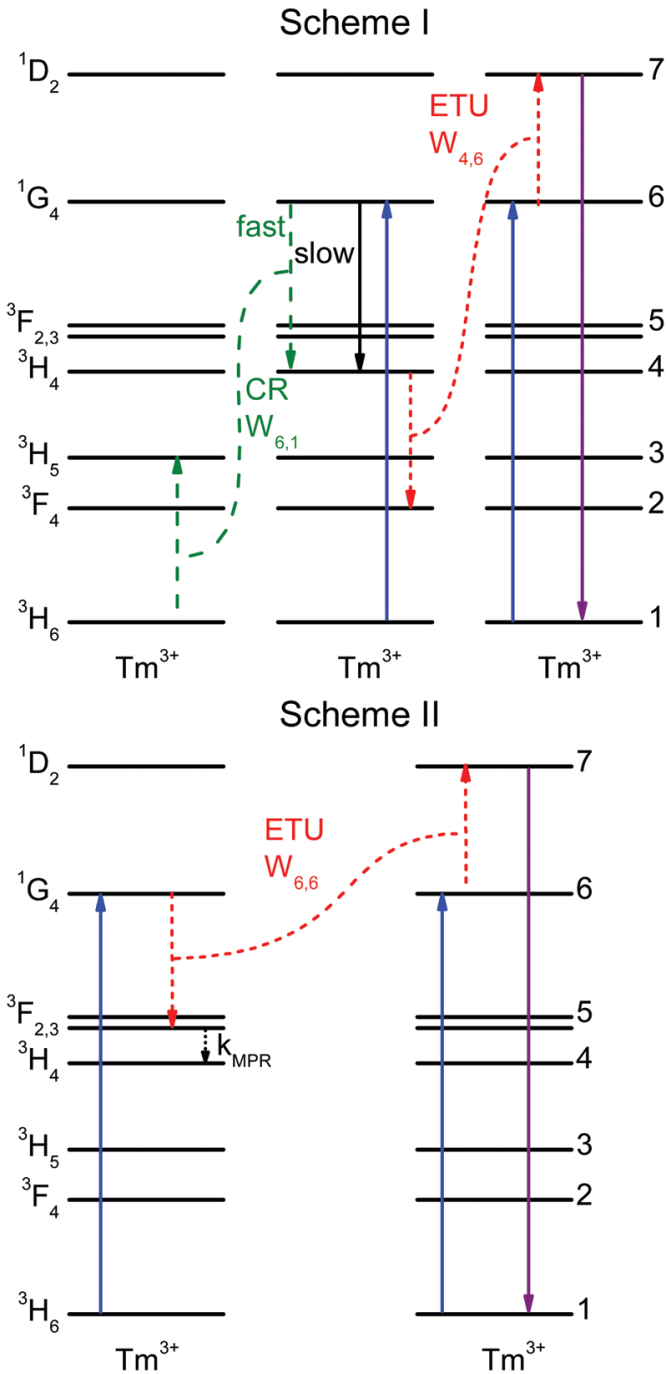

Fig. 4 Possible mechanisms for ${ }^{1} D_{2}$ upconversion in $\mathrm{Tm}^{3+}$. The blue arrow ${ }^{3} \mathrm{H}_{6} \rightarrow{ }^{1} \mathrm{G}_{4}$ indicates the excitation and the violet arrow shows the ${ }^{1} D_{2} \rightarrow{ }^{3} \mathrm{H}_{6}$ upconverted UV emission. The $W_{i, j}$ denote ET processes with initial states $i$ and $j$. Scheme I: two ET processes populate the ${ }^{3} \mathrm{H}_{4}$ and ${ }^{1} \mathrm{D}_{2}$ states. The ${ }^{1} \mathrm{G}_{4} \rightarrow{ }^{3} \mathrm{H}_{4}$ radiative transition (black arrow, slow) and the CR step (green arrows, fast) populate the ${ }^{3} \mathrm{H}_{4}$ state. The ETU step (red) populates the ${ }^{1} D_{2}$ state. Scheme II: one ETU process populates the ${ }^{1} D_{2}$ state. Rapid multiphonon relaxation (MPR) from ${ }^{3} \mathrm{~F}_{3}$ populates the ${ }^{3} \mathrm{H}_{4}$ state.

transition is stronger (by a factor of 2.5 ) in the $0.3 \% \mathrm{Tm}^{3+}$ sample as a result of the CR process that populates the ${ }^{3} \mathrm{H}_{4}$ state. This process depends on the $\mathrm{Tm}^{3+}$ concentration and hence it is stronger for the $0.3 \% \mathrm{Tm}^{3+}$ sample. The ${ }^{1} \mathrm{G}_{4} \rightarrow{ }^{3} \mathrm{H}_{4}$ transition, however, is weaker (by a factor of 2 ) in the $0.3 \% \mathrm{Tm}^{3+}$ sample while other transitions from the ${ }^{1} \mathrm{G}_{4}$ state are not. It is possible that radiative energy transfer ${ }^{1} \mathrm{G}_{4}+{ }^{3} \mathrm{H}_{6} \rightarrow{ }^{3} \mathrm{H}_{4}+{ }^{3} \mathrm{H}_{5}$ plays a role in the higher doped sample, whereby a $\mathrm{Tm}^{3+}$ ion in the ground state absorbs a photon emitted from the ${ }^{1} \mathrm{G}_{4} \rightarrow{ }^{3} \mathrm{H}_{4}$ decay in another $\mathrm{Tm}^{3+}$ ion. Unfortunately, it is not possible to reliably measure the ${ }^{3} \mathrm{H}_{5} \rightarrow{ }^{3} \mathrm{H}_{6}$ and ${ }^{3} \mathrm{~F}_{4} \rightarrow{ }^{3} \mathrm{H}_{6}$ emissions (see below).

Scheme II: the ETU process ${ }^{1} \mathrm{G}_{4}+{ }^{1} \mathrm{G}_{4} \rightarrow{ }^{3} \mathrm{~F}_{3}+{ }^{1} \mathrm{D}_{2}$ can populate the ${ }^{1} \mathrm{D}_{2}$ state rapidly and fast multiphonon relaxation from ${ }^{3} \mathrm{~F}_{3}$ to ${ }^{3} \mathrm{H}_{4}$ can explain the fast rise time of the ${ }^{3} \mathrm{H}_{4}$ state.
The different emission intensities of the ${ }^{3} \mathrm{H}_{4} \rightarrow{ }^{3} \mathrm{H}_{6}$ and ${ }^{1} \mathrm{G}_{4} \rightarrow{ }^{3} \mathrm{H}_{4}$ transitions are explained in the same way as in scheme I.

The width of the emission bands in this lattice made it impossible to measure the decay curve of the ${ }^{3} \mathrm{H}_{5} \rightarrow{ }^{3} \mathrm{H}_{6}$ transition, as it is masked by the ${ }^{1} \mathrm{G}_{4} \rightarrow{ }^{3} \mathrm{H}_{4}$ emission; the ${ }^{3} \mathrm{~F}_{4} \rightarrow{ }^{3} \mathrm{H}_{6}$ emission is at the edge of the detector sensitivity and therefore cannot be measured reliably. The ${ }^{3} \mathrm{H}_{5} \rightarrow{ }^{3} \mathrm{H}_{6}$ decay curve could offer valuable information to decide which of the two schemes prevails.

In order to discover which mechanism explains the experimental data better, we proceed to analyze the data in view of three models: the Inokuti-Hirayama model, an average rate equation model, and a microscopic rate equation model that takes the correct extent of energy transfer into account.

\subsection{The Inokuti-Hirayama}

The samples of $\beta-\mathrm{NaYF}_{4}: \mathrm{Tm}^{3+}$ contain only one optically active ion. In this case the sensitizer in the Inokuti-Hirayama model corresponds to the $\mathrm{Tm}^{3+}$ ions excited in the ${ }^{1} \mathrm{G}_{4}$ state, while the activator are those ions in the ground state.

The ${ }^{1} \mathrm{G}_{4} \rightarrow{ }^{3} \mathrm{~F}_{4}$ decay has been fitted to the Inokuti-Hirayama model according to eqn (2), for the three different interaction multipolarities $n=6$ (d-d), $n=8(\mathrm{~d}-\mathrm{q})$, and $n=10$ (q-q), see Fig. 5 and Table S1, ESI. $\dagger$ All fits are in good agreement with the experimental data, the best being that for dipole-dipole interaction. It is possible that all multipolarities play a role in the energy transfer process.

In order to determine the interaction critical radius using eqn (3), the activator concentration $c_{\mathrm{A}}$ has to be obtained. $c_{\mathrm{A}}$ is the concentration of $\mathrm{Tm}^{3+}$ ions in the ground state, and is related to the fraction $F_{\mathrm{E}}$ of excited $\mathrm{Tm}^{3+}$ ions in the ${ }^{1} \mathrm{G}_{4}$ state right after the excitation pulse: $c_{\mathrm{A}}=\left(1-F_{\mathrm{E}}\right) c_{\mathrm{Tm}^{3+}}$, where $c_{\mathrm{Tm}^{3+}}$ is the concentration of $\mathrm{Tm}^{3+}$ ions in the sample, $c_{\mathrm{Tm}^{3+}}=4.14 \times$ $10^{19}$ ions $\mathrm{cm}^{-3}$ for $\beta-\mathrm{NaYF}_{4}: 0.3 \% \mathrm{Tm}^{3+} . F_{\mathrm{E}}$ can be estimated

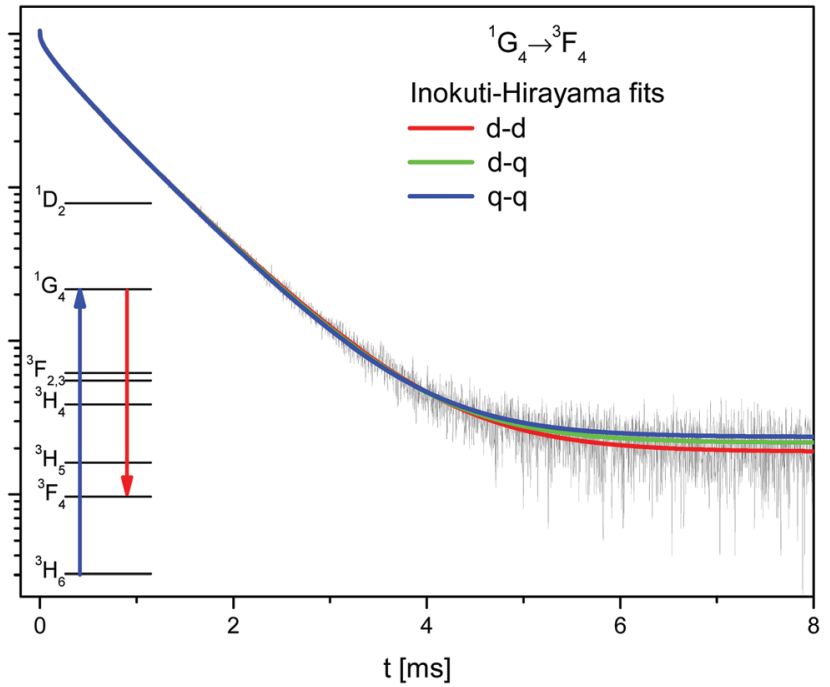

Fig. 5 Decay of the ${ }^{1} \mathrm{G}_{4}$ state (black line) in $\beta-\mathrm{NaYF}_{4}: 0.3 \% \mathrm{Tm}^{3+}$ with the Inokuti-Hirayama fit for dipole-dipole $(d-d)$, dipole-quadrupole $(d-q)$, and quadrupole-quadrupole $(q-q)$ interaction mechanisms. 
from the absorption transition probability $R_{\mathrm{P}}$ and the pulse duration $\Delta t$. The absorption probability is given by

$$
R_{\mathrm{P}}=\frac{P}{A_{\mathrm{b}}} \sigma \frac{1}{h \nu}
$$

with $P$ being the excitation power, $A_{\mathrm{b}}$ the excitation beam area, $\sigma$ the absorption cross-section, and $h \nu$ the photon energy. ${ }^{32-34}$

The absorption cross-section for ${ }^{3} \mathrm{H}_{6} \rightarrow{ }^{1} \mathrm{G}_{4}$ is $\sigma=7.77 \times$ $10^{-22} \mathrm{~cm}^{2}$, see Fig. S6, ESI. $\dagger$ For the laser used in these experiments the relevant parameters are $P=6 \times 10^{6} \mathrm{~W}$, $A_{\mathrm{b}}=3 \times 10^{-2} \mathrm{~cm}^{2}$, and $\Delta t=5 \mathrm{~ns}$, which results in $R_{\mathrm{P}}=$ $3.67 \times 10^{5} \mathrm{~s}^{-1}$ and $F_{\mathrm{E}} \approx R_{\mathrm{P}} \Delta t \approx 0.2 \%$. Therefore, most $\mathrm{Tm}^{3+}$ ions remain in the ground state after the pulse and $c_{\mathrm{A}} \approx c_{\mathrm{Tm}^{3+}}$.

Using the values determined above and eqn (3), the critical radius for the d-d CR process is $R_{\mathrm{c}, \mathrm{IH}}=11.8 \pm 0.1 \AA$. This value is similar to those found in other lattices for other $\mathrm{Tm}^{3+}$ interactions. ${ }^{31,35,36}$

The Inokuti-Hirayama model agrees well with the experimental data due to the low concentration of $\mathrm{Tm}^{3+}$ ions. The long distances between $\mathrm{Tm}^{3+}$ ions restrict the extent of energy migration. The ${ }^{1} \mathrm{G}_{4}$ decay has also been fitted using the Burshtein, Zusman and Yokota-Tanimoto models, see Fig. S7, ESI. $\dagger$ These models introduce energy migration among the sensitizers in addition to the sensitizer-activator ET. For all these models the fit results in zero or negligible energy migration rates.

The decay curve is therefore affected only by the energy transfer to nearby $\mathrm{Tm}^{3+}$ ions and the intrinsic radiative decay of the ${ }^{1} \mathrm{G}_{4}$ state. Although the Inokuti-Hirayama model correctly predicts the ${ }^{1} \mathrm{G}_{4}$ decay curve and shows that energy migration is absent, it does not make any predictions about the ${ }^{3} \mathrm{H}_{4}$ and ${ }^{1} \mathrm{D}_{2}$ decay curves or the specific ET processes (ETU and/or CR) that take place. A rate equation model can give information about the decay curves of all states and the ET processes involved.

\subsection{Average rate equation model}

The excitation, emission, CR, and ETU processes can be modeled with the help of a rate equation system. ${ }^{26,37}$ This is a system of $n$ differential equations that depend on $n$ variables (the energy state populations) and the time $t$. In this case $n=7$, as the states higher in energy than ${ }^{1} \mathrm{D}_{2}$ do not participate in the mechanism, see Fig. 4 . The population of state $i(i=1, \ldots, 7)$ is denoted by $y_{i}$, e.g. the population of the ground state is $y_{1}$.

The absorption and emission processes shown in Fig. 1 and the energy transfer processes depicted in Fig. 4 are expressed mathematically in eqn (6). The absorption of light corresponding to the ${ }^{3} \mathrm{H}_{6} \rightarrow{ }^{1} \mathrm{G}_{4}$ transition is modeled with $R_{\mathrm{P}}\left(y_{1}-13 / 9 y_{6}\right)$, where $R_{\mathrm{P}}$ is the absorption probability, see eqn (5). The first term in the parentheses refers to stimulated absorption and the second to stimulated emission (13/9 is the relative degeneracy of the initial and final states). The decay is modeled with a decay rate constant $k_{i}$ that includes the radiative and non-radiative contributions. The emission from state $i$ to state $f$ is modeled with a branching ratio parameter $\beta_{i, f}$. For example, for the ${ }^{3} \mathrm{H}_{5}$ state the term $\beta_{6,3} k_{6} y_{6}$ (with $\beta_{6,3}<1$ ) indicates that a fraction of the decay from state $6\left({ }^{1} \mathrm{G}_{4}\right)$ populates state $3\left({ }^{3} \mathrm{H}_{5}\right)$. Energy transfer steps with initial states $i$ and $j$ are modeled with $W_{i, j} y_{i} y_{j}$, where $W_{i, j}$ is a constant to be fitted using experimental data. Eqn (6) shows the state populations in red to help distinguish the linear and non-linear terms.

$$
\begin{aligned}
\frac{\mathrm{d} y_{7}}{\mathrm{~d} t}= & -k_{7} y_{7}+W_{4,6} y_{4} y_{6}+W_{6,6} y_{6} y_{6} \\
\frac{\mathrm{d} y_{6}}{\mathrm{~d} t}= & -k_{6} y_{6}+R_{\mathrm{P}}\left(y_{1}-13 / 9 y_{6}\right) \\
& -W_{4,6} y_{4} y_{6}-W_{6,1} y_{6} y_{1}-2 W_{6,6} y_{6} y_{6} \\
\frac{\mathrm{d} y_{5}}{\mathrm{~d} t}= & -k_{5} y_{5}+W_{6,6} y_{6} y_{6} \\
\frac{\mathrm{d} y_{4}}{\mathrm{~d} t}= & -k_{4} y_{4}+\beta_{6,4} k_{6} y_{6}+\beta_{5,4} k_{5} y_{5}-W_{4,6} y_{4} y_{6}+W_{6,1} y_{6} y_{1} \\
\frac{\mathrm{d} y_{3}}{\mathrm{~d} t}= & -k_{3} y_{3}+\beta_{6,3} k_{6} y_{6}+W_{6,1} y_{6} y_{1} \\
\frac{\mathrm{d} y_{2}}{\mathrm{~d} t}= & -k_{2} y_{2}+\beta_{7,2} k_{7} y_{7}+\beta_{6,2} k_{6} y_{6} \\
& +\beta_{4,2} k_{4} y_{4}+\beta_{3,2} k_{3} y_{3}+W_{4,6} y_{4} y_{6} \\
\frac{\mathrm{d} y_{1}}{\mathrm{~d} t}= & -\left(\frac{\mathrm{d} y_{2}}{\mathrm{~d} t}+\frac{\mathrm{d} y_{3}}{\mathrm{~d} t}+\frac{\mathrm{d} y_{4}}{\mathrm{~d} t}+\frac{\mathrm{d} y_{5}}{\mathrm{~d} t}+\frac{\mathrm{d} y_{6}}{\mathrm{~d} t}+\frac{\mathrm{d} y_{7}}{\mathrm{~d} t}\right)
\end{aligned}
$$

The initial conditions are described by the ground state population set to one and the others to zero, $y(t=0)=(1,0,0,0,0,0,0)$. The values of the decay rates and branching ratios are constants determined from decay and emission experiments in $\beta-\mathrm{NaYF}_{4}: 0.01 \% \mathrm{Tm}^{3+}$, and are summarized in Table 1 . These values are in good agreement with Judd-Ofelt calculations on $\beta$-NaGdF $4: \mathrm{Tm}^{3+} \cdot{ }^{38}$ The lifetimes of the ${ }^{3} \mathrm{~F}_{4},{ }^{3} \mathrm{H}_{4},{ }^{1} \mathrm{G}_{4}$, and ${ }^{1} \mathrm{D}_{2}$ states are dominated by their radiative decay in this low phonon energy host lattice. The ${ }^{3} \mathrm{H}_{5}$ and ${ }^{3} \mathrm{~F}_{3}$ states have shorter lifetimes due to multiphonon decay across the rather small energy gaps towards the ${ }^{3} \mathrm{~F}_{4}$ and ${ }^{3} \mathrm{H}_{4}$ states, respectively.

The ${ }^{3} \mathrm{~F}_{3}$ state decays mainly to the ${ }^{3} \mathrm{H}_{4}$ state, so the decay rate $k_{5}$ is the multiphonon relaxation rate $k_{\mathrm{MPR}}$ shown in Fig. 4 .

The solution to the system of equations is the population of each state as a function of time. The free parameters are the CR

Table 1 Experimentally determined constants for all rate equation simulations. These parameters were obtained from decay curves and emission spectra of $\beta-\mathrm{NaYF}_{4}: 0.01 \% \mathrm{Tm}^{3+}$. For each state, the branching ratio $\beta$ to the ground state is one minus the sum of all other branching ratios

\begin{tabular}{llll}
\hline $\mathrm{Tm}^{3+}$ state & No. & Lifetime & $\beta_{i, f}$ (final state) \\
\hline${ }^{1} \mathrm{D}_{2}$ & 7 & $67.5 \mu \mathrm{s}$ & $0.40\left({ }^{3} \mathrm{~F}_{4}\right)$ \\
${ }^{1} \mathrm{G}_{4}$ & & $760 \mu \mathrm{s}$ & $0.09\left({ }^{3} \mathrm{~F}_{3}\right)$ \\
& 6 & & $0.07\left({ }^{3} \mathrm{~F}_{4}\right)$ \\
${ }^{3} \mathrm{~F}_{3}$ & & $20 \mu \mathrm{s}$ & $0.22\left({ }^{3} \mathrm{H}_{5}\right)$ \\
${ }^{3} \mathrm{H}_{4}$ & 5 & $1.9 \mathrm{~ms}$ & $0.999\left({ }^{3} \mathrm{H}_{4}\right)$ \\
& 4 & & $0.3\left({ }^{3} \mathrm{~F}_{4}\right)$ \\
${ }^{3} \mathrm{H}_{5}$ & 3 & $29 \mu \mathrm{s}$ & $0.06\left({ }^{3} \mathrm{H}_{5}\right)$ \\
${ }^{3} \mathrm{~F}_{4}$ & 2 & $12 \mathrm{~ms}$ & - \\
${ }^{3} \mathrm{H}_{6}$ & 1 & - & -
\end{tabular}

${ }^{a}$ The $k_{5}$ decay rate is optimized in the simulation of scheme II. 
a)

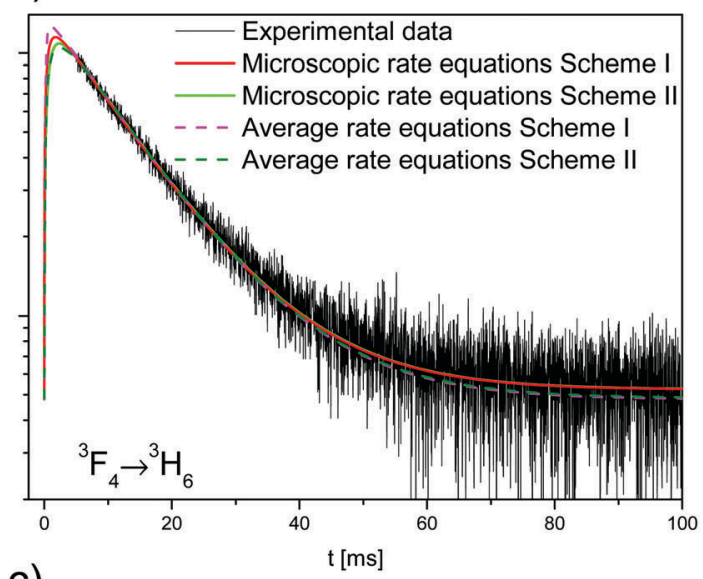

c)

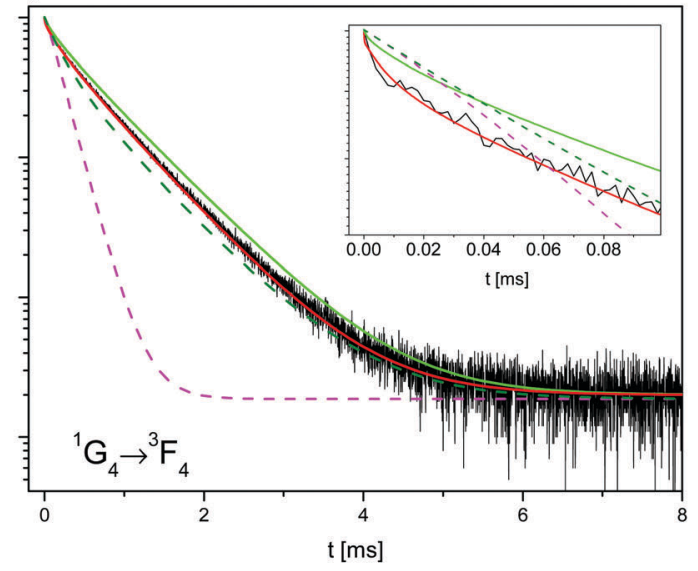

b)

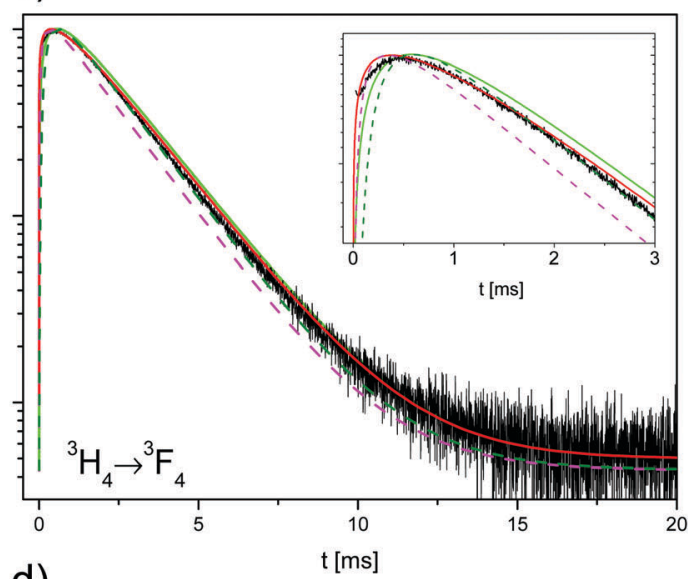

d)

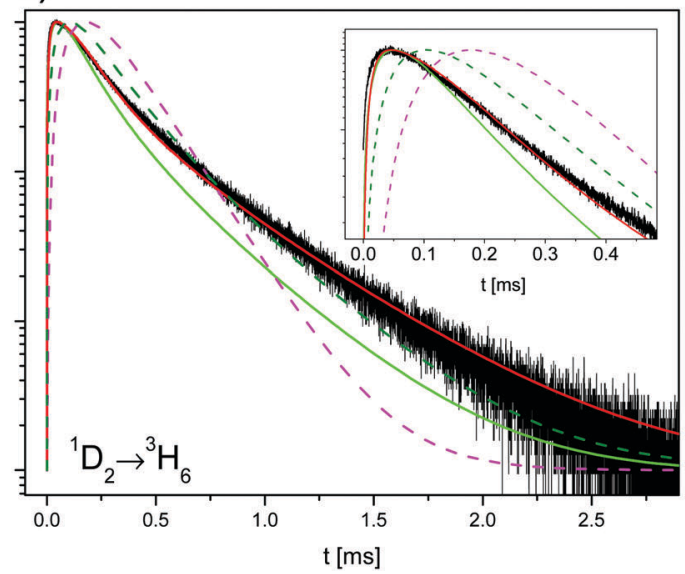

Fig. 6 Experimental data and calculated decay curves. (a) ${ }^{3} \mathrm{~F}_{4}$, (b) ${ }^{3} \mathrm{H}_{4}$, (c) ${ }^{1} \mathrm{G}_{4}$, and (d) ${ }^{1} \mathrm{D}_{2}$ state decays. The experimental data are plotted as black lines, the fits to the average rate equation model as dashed lines (scheme I, magenta; scheme II, olive), and the fits to the microscopic rate equation model as full lines (scheme I, red; scheme II, green). The insets show the decay at the short time scale.

rate $W_{6,1}$ and the ETU rate $W_{4,6}$ (with $W_{6,6}=0$ ) for scheme I and the ETU rate $W_{6,6}$ (with $W_{6,1}=W_{4,6}=0$ ) and the decay rate $k_{5}$ for scheme II. The parameters for both schemes were fitted to the experimental data and are shown as dashed lines in Fig. 6. The best fit values of the parameters for scheme I are $W_{6,1}=$ $2.13 \times 10^{3} \mathrm{~s}^{-1}$ (CR rate) and $W_{4,6}=1.10 \times 10^{8} \mathrm{~s}^{-1}$ (ETU rate), whereas they are $W_{6,6}=1.58 \times 10^{7} \mathrm{~s}^{-1}$ and $k_{\mathrm{MPR}}=1.00 \times 10^{6} \mathrm{~s}^{-1}$ for scheme II.

Neither fit to the average rate equation model is able to correctly reproduce the decay of the ${ }^{1} \mathrm{G}_{4}$ and ${ }^{1} \mathrm{D}_{2}$ states, especially for scheme I. The reason for this strong discrepancy with the experimental data is the absence of energy migration in the samples. There are two classes of excited ions: isolated ions that decay radiatively to the ground state and those that transfer their energy to another ion. Most ions belong to the former group, and hence the measured lifetime of the ${ }^{1} \mathrm{G}_{4}$ decay $(719 \mu \mathrm{s})$ is very similar to the intrinsic single exponential decay $(760 \mu \mathrm{s})$. Ions in the latter group have a much faster decay rate as they efficiently transfer their energy.

The average rate equation model assumes infinitely fast energy migration among all ions in the sample so that the population of each state does not deviate much from the average. ${ }^{26,28}$ For these samples however, as previously demonstrated, energy migration is negligible. A more sophisticated microscopic rate equation model that can predict the decay curves of all states and correctly takes into account the correct extent of energy transfer is presented in the next subsection.

\subsection{Microscopic rate equation model}

A $\beta-\mathrm{NaYF}_{4}: 0.3 \% \mathrm{Tm}^{3+}$ lattice with $50 \times 50 \times 50$ unit cells is simulated and the distances between all ions are calculated, up to $d_{\max }=150 \AA$. Then a rate equation system like eqn (6) is assigned to each $\mathrm{Tm}^{3+}$ ion in the lattice. For a concentration of $0.3 \%$ this results in $571 \mathrm{Tm}^{3+}$ ions in the simulation. The ET rates are calculated taking into account the distances to the other ions and the chosen multipolarity of the interaction with eqn (1). These $W_{i, j}$ rates are not average ET parameters but actually the $C_{\mathrm{SA}}$ rates in eqn (1). The resulting nonlinear system of differential equations has 3997 variables, corresponding to 7 energy states for 571 ions. Its solution is the population of each state as a function of time. In order to compare the model with the experimental data, the average of each state is calculated across all ions. ${ }^{16} \mathrm{~A}$ fit of the parameters to the experimental data is performed. 
Table 2 Fixed parameters for the energy migration microscopic rate equation model

\begin{tabular}{ll}
\hline Fixed parameter & Value \\
\hline$a$ & $5.9734(6) \AA$ \\
$c$ & $3.5296(4) \AA$ \\
$N_{\text {cells }}$ & 125000 \\
$d_{\max }(0.3 \mathrm{~mol} \%)$ & $150 \AA$ \\
$c_{\text {Tm }}\left(0.14 \times 10^{19} \mathrm{ions} \mathrm{cm}^{-3}\right.$ \\
$\sigma\left(\nu=21100 \mathrm{~cm}^{-1}\right)$ & $4.14 \times 10^{-22} \mathrm{~cm}^{2}$ \\
$P$ & $7.77 \times 10^{7} \mathrm{Cm}^{-2}$ \\
$A_{\mathrm{b}}$ & $1.7 \times 10^{\circ}$ \\
$\Delta t_{\text {pulse }}$ & $5 \mathrm{~ns}$ \\
\hline
\end{tabular}

The fit normalized root-mean-square deviation $\varepsilon_{\text {total }}$ is calculated by

$$
\varepsilon_{\text {total }}=\sqrt{\sum_{\text {state }} \varepsilon_{\text {state }}^{2}}
$$

where the normalized root-mean-square deviation $\varepsilon_{\text {state }}$ of each state is calculated by

$$
\varepsilon_{\text {state }}=\frac{1}{\left\langle N_{\text {state, avg }}\right\rangle} \sqrt{\frac{1}{N_{t, \exp }} \sum_{t=0}^{t_{\mathrm{f}}}\left(N_{\text {state, avg }}(t)-N_{\text {state, } \exp }(t)\right)^{2}}
$$

where $N_{\text {state,avg }}(t)$ is the simulated average population of the state, $N_{\text {state,exp }}(t)$ is the experimental decay curve, and $N_{t, \text { exp }}$ is the number of experimental data points.

The values of the fixed parameters are given in Table 2 and the values of the lifetimes and branching ratios are the same as for the average rate equation model given in Table 1.

The results of the simulation are compared to the experimental data in Fig. 6. Scheme I (red lines) fits the experimental data better than scheme II (green lines), with a normalized rootmean-square deviation of $\varepsilon_{\mathrm{I}}=0.49$ versus $\varepsilon_{\mathrm{II}}=0.68$.

For scheme I, the fitted values are $W_{4,6}=1.258 \times 10^{-38} \mathrm{~cm}^{6} \mathrm{~s}^{-1}$ (d-d interaction) for the ETU and $W_{6,1}=4.205 \times 10^{-39} \mathrm{~cm}^{6} \mathrm{~s}^{-1}$ (d-d interaction) for the CR. The corresponding critical radii are $R_{\mathrm{c}, \mathrm{ETU}}=16.9 \AA$ and $R_{\mathrm{c}, \mathrm{CR}}=12.1 \AA$. The agreement between experiment and simulation is excellent. The simulation correctly reproduces all decay curves, especially the rise of the ${ }^{3} \mathrm{H}_{4}$ and ${ }^{1} \mathrm{D}_{2}$ states, the non-exponential decay of the ${ }^{1} \mathrm{D}_{2}$ state, and the shape of the ${ }^{1} \mathrm{G}_{4}$ curve at the short timescale. For both ET steps, the dipole-dipole interaction $(n=6)$ gives the best fit to the experimental data, see Table S2, ESI. $\dagger$

The critical radius of $R_{\mathrm{c}, \mathrm{CR}}=12.1 \AA$ for the CR process is very similar to $R_{\mathrm{c}, \mathrm{IH}}=11.8 \pm 0.1 \AA$ for the Inokuti-Hirayama fit. This can be understood since the CR process is a transfer to an ion in its ground state, precisely the only kind of ET process that the Inokuti-Hirayama model deals with. The population of the ${ }^{1} \mathrm{D}_{2}$ state is small and the effect of the ETU on the ${ }^{1} \mathrm{G}_{4}$ decay curve is negligible, so the simple Inokuti-Hirayama fit is not much affected by it. However, both ET processes contribute to the decay curve of the ${ }^{3} \mathrm{H}_{4}$ state.

For scheme II, the best fit for the ETU rate is $W_{6,6}=1.60 \times$ $10^{-35} \mathrm{~cm}^{6} \mathrm{~s}^{-1}$ with multipolarity $n=6(\mathrm{~d}-\mathrm{d})$ and ${ }^{3} \mathrm{~F}_{3}$ decay rate $k_{\mathrm{MPR}}=3.26 \times 10^{8} \mathrm{~s}^{-1}$. The corresponding critical radius is

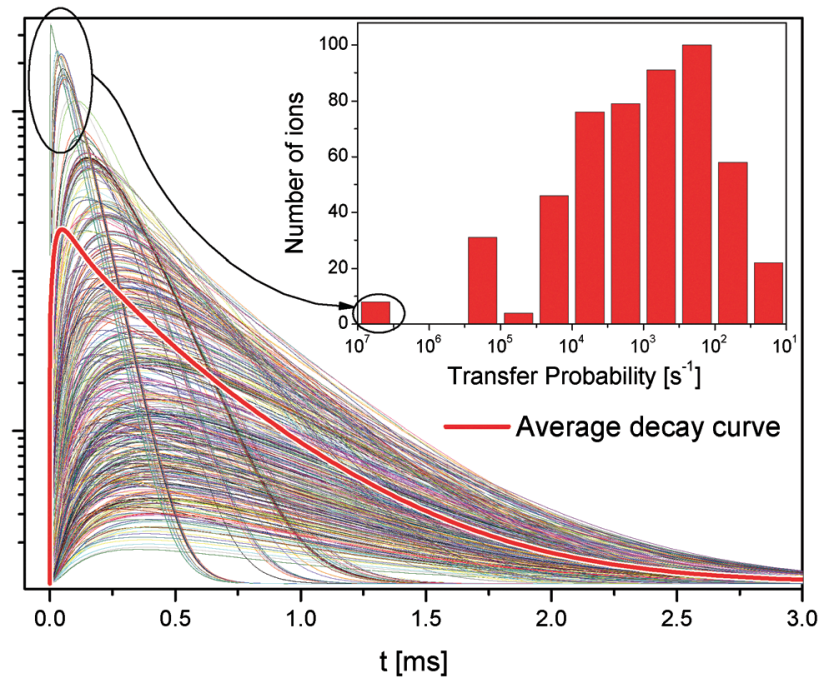

Fig. 7 All ${ }^{1} D_{2}$ simulated decay curves of the $571 \mathrm{Tm}^{3+}$ ions in a $\beta-\mathrm{NaYF}_{4}: 0.3 \% \mathrm{Tm}^{3+}$ sample of $50 \times 50 \times 50$ unit cells. The average population is shown as a thick red curve. The inset shows the histogram of the number of ions with a given energy transfer probability to its nearest neighbor. The ions with a higher transfer probability have faster rise and decay times.

$R_{\mathrm{c}, \mathrm{ETU}}=47.9 \AA$, a value that is much larger than the usual range of 5-20 А. ${ }^{31,35,36}$ Fischer et al. have estimated the energy gap law parameters for this lattice. ${ }^{37}$ Using a conservative estimate of the energy difference between the ${ }^{3} \mathrm{H}_{4}$ and ${ }^{3} \mathrm{~F}_{3}$ states of $\Delta E=1800 \mathrm{~cm}^{-1}$ the estimated rate is $k_{\mathrm{MPR}}=4.61 \times 10^{4} \mathrm{~s}^{-1}$, several orders of magnitude smaller than the fitted value of $3.26 \times 10^{8} \mathrm{~s}^{-1}$. The magnitudes of the optimized parameters for scheme II are physically unreasonable. Together with the higher fit error, it is concluded that scheme II cannot explain the experimental data correctly.

For either scheme, including energy migration among the $\mathrm{Tm}^{3+}$ ions does not improve the fit, in agreement with the results in Section 4.1.

4.3.1 Microscopic analysis of the decay curves. The microscopic rate equation model is able to explain why the ${ }^{1} \mathrm{D}_{2}$ decay curve is not single exponential, see Fig. 7 . At this low dopant concentration, the average minimum distance between $\mathrm{Tm}^{3+}$ ions is about $15 \AA ;^{16}$ however, some ions are as close as $3.53 \AA$, the minimum distance in this lattice.

The transfer probability depends on the inverse sixth power of the distance, see eqn (1); this means that small differences in the distance have a large effect on the transfer probability, see the inset in Fig. 7.

Fig. 7 shows all ${ }^{1} \mathrm{D}_{2}$ state decay curves from the $571 \mathrm{Tm}^{3+}$ ions in the simulated lattice introduced above. The average decay curve is shown as a thick red line and is the same as in Fig. 6d.

Some ions reach the ${ }^{1} \mathrm{D}_{2}$ state much faster than others and with a much higher probability. Those ions have neighbors at the nearest possible distance, and therefore interact very strongly; their decay curves dominate the average decay curve at the short timescale. Some other ions have neighbors further away and become excited at later times; they dominate the 
average decay curve at longer times. Because of the discrete nature of the distances in the lattice, the ET probability between two ions drops from about $6 \times 10^{6} \mathrm{~s}^{-1}$ to $3 \times 10^{5} \mathrm{~s}^{-1}$ for separations of $3.53 \AA$ and $5.9 \AA$, respectively.

The features in the decay curves of other states have similar microscopic origins; however, since the ${ }^{1} \mathrm{D}_{2}$ population requires two ET steps, this state is more strongly affected than others by the lattice distance distribution.

\section{Conclusions}

Two $\mathrm{Tm}^{3+}-\mathrm{Tm}^{3+}$ energy transfer processes are responsible for the upconverted UV luminescence in $\beta-\mathrm{NaYF}_{4}: \mathrm{Tm}^{3+}$ after ${ }^{3} \mathrm{H}_{6} \rightarrow{ }^{1} \mathrm{G}_{4}$ blue excitation at $21140 \mathrm{~cm}^{-1}$ : the cross-relaxation step ${ }^{3} \mathrm{H}_{6}+{ }^{1} \mathrm{G}_{4} \rightarrow{ }^{3} \mathrm{H}_{5}+{ }^{3} \mathrm{H}_{4}$ and the energy transfer upconversion step ${ }^{3} \mathrm{H}_{4}+{ }^{1} \mathrm{G}_{4} \rightarrow{ }^{3} \mathrm{~F}_{4}+{ }^{1} \mathrm{D}_{2}$. The alternative mechanism consisting of the energy transfer upconversion step ${ }^{1} \mathrm{G}_{4}+{ }^{1} \mathrm{G}_{4} \rightarrow{ }^{3} \mathrm{~F}_{3}+{ }^{1} \mathrm{D}_{2}$ and non-radiative relaxation ${ }^{3} \mathrm{~F}_{3} \rightarrow{ }^{3} \mathrm{H}_{4}$ cannot explain all experimental data. It requires unreasonable parameters for the upconversion and multiphonon relaxation rates.

The interaction strength and multipolarity have been determined for each process from a fit to experimental data. All results indicate that energy migration is negligible in these samples and that all energy transfer processes are due to dipole-dipole interactions.

The microscopic rate equation model accurately fits the experimental data and offers a detailed view on the ion to ion energy transfer processes. This microscopic model is a significant improvement on other models such as Inokuti-Hirayama and the average rate equation models because it takes into account the distances between ions and all decay and energy transfer processes in the sample, including energy migration.

\section{Acknowledgements}

The financial support of the EU FP7 ITN LUMINET (Grant agreement No. 316906) is gratefully acknowledged.

\section{References}

1 F. Auzel, Chem. Rev., 2004, 104, 139-174.

2 A. Gharavi and G. L. McPherson, Chem. Phys. Lett., 1992, 200, 279-282.

3 H. Yang and J. Gao, Phys. B, 2013, 426, 31-34.

4 E. Cantelar, G. Torchia and F. Cussó, J. Lumin., 2007, 122-123, 459-462.

5 G. Özen, A. Kermaoui, J. Denis, X. Wu, F. Pelle and B. Blanzat, J. Lumin., 1995, 63, 85-96.

6 R. J. Thrash and L. F. Johnson, J. Opt. Soc. Am. B, 1994, 11, 881-885.

7 M. A. Noginov, M. Curley, P. Venkateswarlu, A. Williams and H. P. Jenssen, J. Opt. Soc. Am. B, 1997, 14, 2126-2136.

8 X. X. Zhang, P. Hong, M. Bass and B. H. T. Chai, Phys. Rev. B: Condens. Matter Mater. Phys., 1995, 51, 9298-9301.

9 J. Zhou, Q. Liu, W. Feng, Y. Sun and F. Li, Chem. Rev., 2015, 115, 395-465.
10 A. Stepuk, D. Mohn, R. N. Grass, M. Zehnder, K. W. Krämer, F. Pellé, A. Ferrier and W. J. Stark, Dent. Mater., 2012, 28, 304-311.

11 J. C. Goldschmidt and S. Fischer, Adv. Opt. Mater., 2015, 3, 510-535.

12 W. Yang, X. Li, D. Chi, H. Zhang and X. Liu, Nanotechnology, 2014, 25, 482001.

13 Y. Chen, S. Mishra, G. Ledoux, E. Jeanneau, M. Daniel, J. Zhang and S. Daniele, Chem. - Asian J., 2014, 9, 2415-2421.

14 O. Malta, J. Non-Cryst. Solids, 2008, 354, 4770-4776.

15 A. Aebischer, M. Hostettler, J. Hauser, K. Krämer, T. Weber, H. U. Güdel and H.-B. Bürgi, Angew. Chem., Int. Ed., 2006, 45, 2802-2806.

16 P. Villanueva-Delgado, K. W. Krämer and R. Valiente, J. Phys. Chem. C, 2015, 119, 23648-23657.

17 M. Quintanilla, E. Cantelar, F. Cussó, J. A. Barreda-Argüeso, J. González, R. Valiente and F. Rodríguez, Opt. Mater. Express, 2015, 5, 1168-1182.

18 T. Kushida, J. Phys. Soc. Jpn., 1973, 34, 1318-1326.

19 M. Inokuti and F. Hirayama, J. Chem. Phys., 1965, 43, 1978-1989.

20 L. Zusman, J. Exp. Theor. Phys., 1977, 46, 347-351.

21 M. Artamonova, C. M. Briskina, A. Burshtein, L. Zusman and A. Skleznev, J. Exp. Theor. Phys., 1972, 35, 457-461.

22 A. Burshtein, J. Exp. Theor. Phys., 1972, 35, 882-885.

23 A. Burshtein, J. Lumin., 1985, 34, 167-188.

24 M. Yokota and O. Tanimoto, J. Phys. Soc. Jpn., 1967, 22, 779-784.

25 I. R. Martín, V. D. Rodríguez, U. R. Rodríguez-Mendoza, V. Lavín, E. Montoya and D. Jaque, J. Chem. Phys., 1999, 111, 1191-1194.

26 W. Grant, Phys. Rev. B: Solid State, 1971, 4, 648-663.

27 D. A. Zubenko, M. A. Noginov, V. A. Smirnov and I. A. Shcherbakov, Phys. Rev. B: Condens. Matter Mater. Phys., 1997, 55, 8881-8886.

28 L. Agazzi, K. Wörhoff and M. Pollnau, J. Phys. Chem. C, 2013, 117, 6759-6776.

29 K. W. Krämer, D. Biner, G. Frei, H. U. Güdel, M. P. Hehlen and S. R. Lüthi, Chem. Mater., 2004, 16, 1244-1251.

30 G. H. Dieke and H. Crosswhite, Spectra and Energy Levels of Rare Earth Ions in Crystals, Interscience Publishers, 1968.

31 D.-C. Yu, R. Martín-Rodríguez, Q.-Y. Zhang, A. Meijerink and F. T. Rabouw, Light: Sci. Appl., 2015, 4, e344.

32 A. Siegman, Lasers, University Science Books, Sausalito, California, 1986.

33 W. Demtröder, Laser Spectroscopy: Basic Principles, Springer, Berlin, Heidelberg, 2008, vol. 1.

34 D. R. Gamelin and H. U. Güdel, in Upconversion Processes in Transition Metal and Rare Earth Metal Systems, ed. H. Yersin, Springer, Berlin, Heidelberg, 2001, pp. 1-56.

35 S. Guy, M. Malinowski, Z. Frukacz, M. Joubert and B. Jacquier, J. Lumin., 1996, 68, 115-127.

36 L. Yan, Z. Xiao, F. Zhu, F. Zhang and A. Huang, J. Opt. Soc. Am. B, 2010, 27, 452-457.

37 S. Fischer, H. Steinkemper, P. Löper, M. Hermle and J. C. Goldschmidt, J. Appl. Phys., 2012, 111, 013109.

38 P. Villanueva-Delgado, D. Biner and K. W. Krämer, J. Lumin., 2016, DOI: 10.1016/j.jlumin.2016.04.023. 